\title{
Corela
}

Cognition, représentation, langage

HS-1 1 | 2012

RJC Cotexte, contexte, situation

\section{Le modèle cognitif et la TOE : deux points de vue sur l'identité sémantique des unités polysémiques}

\section{Olga Kravchenko-Biberson}

\section{OpenEdition}

\section{Journals}

Édition électronique

URL : http://journals.openedition.org/corela/2024

DOI : $10.4000 /$ corela.2024

ISSN : $1638-573 \mathrm{X}$

\section{Éditeur}

Cercle linguistique du Centre et de I'Ouest - CerLICO

\section{Référence électronique}

Olga Kravchenko-Biberson, « Le modèle cognitif et la TOE : deux points de vue sur l'identité sémantique des unités polysémiques », Corela [En ligne], HS-11 | 2012, mis en ligne le 07 novembre 2011, consulté le 01 mai 2019. URL : http://journals.openedition.org/corela/2024 ; DOI : 10.4000/ corela.2024

Ce document a été généré automatiquement le 1 mai 2019.

\section{(i)(2)}

Corela - cognition, représentation, langage est mis à disposition selon les termes de la licence Creative Commons Attribution - Pas d'Utilisation Commerciale - Partage dans les Mêmes Conditions 4.0 International. 


\title{
Le modèle cognitif et la TOE : deux points de vue sur l'identité sémantique des unités polysémiques
}

\author{
Olga Kravchenko-Biberson
}

\section{Les préfixes, les particules et leurs problèmes non résolus.}

1 Les polysèmes, tels que les préfixes et les particules verbales,se présentent à nous comme une source de multiples problèmes et de paradoxes non résolus.

2 Puisque le préfixe est très répandu dans les langues slaves, le russe en l'occurrence, mais que la particule prépositionnelle et adverbiale est typique des langues germaniques auxquelles l'anglais appartient, ces unités seront traitées ici à partir du russe et de l'anglais que nous utiliserons comme métalangues.

3 En premier lieu, ces problèmes concernent leur contenu et leur définition sémantiques. La difficulté réside dans le fait que la diversité des instanciations et l'unité sémantique sous-jacente constituent deux traits caractéristiques apparemment opposés mais complémentaires de la structure de ces éléments productifs du lexique.

4 En effet, si elle privilégie la diversité des effets de sens, la description de leur sémantique risque, inévitablement, de prendre la forme d'une liste énumérant leurs significations distinctes.

5 Cette éventualité s'avère peu satisfaisante car, paradoxalement, même si la distance sémantique entre les différentes instanciations d'un préfixe ou d'une particule peut paraître très forte, la capacité que possède par exemple tout locuteur natif de générer de nouveaux verbes préfixés ou à particule et de les interpréter avec exactitude, témoigne de l'existence d'un lien sémantique entre elles. De plus, il semble évident que si les différents 
sens d'un item polysémique sont liés, c'est bien parce qu'ils possèdent un ou plusieurs traits caractéristiques communs.

6 En outre, le simple inventaire des différents sens ne fait pas avancer l'étude de leur émergence, d'autant plus que les préfixes et les particules ne cessent de créer des verbes avec de nouveaux effets de sens ce qui rend inenvisageable l'établissement d'une liste exhaustive de toutes leurs valeurs distinctes possibles.

7 C'est ainsi que, bien qu'absent dans les dictionnaires consultés, le préfixé réfléchi РАСКАССИРОВАТЬСЯ - raskassirovat'sja (litt.ras-consommer-se) se trouve dans la presse économique :

(1) СУММА КРЕДИТОВ ДОЛЖНА РАСКАССИРОВАТЬСЯ ПО УРОВНЮ ЭФФЕКТИВНОСТИ, ПРОСЧИТЫВАЕМОЙ ПО БИЗНЕС-ПЛАНУ

Summakreditovdolžnaraskassirovat'sja po urovnjueffektivnosti, somme credits $_{\text {Gén. }}$ doit ras-consommer-se selon niveau ${ }_{\text {Dat. }}$ Efficacité ${ }_{\text {Gén. }}$ prosčityvaemoi po biznes-planu.

calculé selon business-plan ${ }_{\text {Dat. }}$

'la somme des crédits doit être consommée en fonction de l'efficacité calculée à partir du business-plan', [Novosti malogo biznesa 15(157) du 19.04.2002] $]^{1}$.

Il s'ensuit donc qu'à l'aide de la polysémisation, les usagers de la langue subviennent aux besoins de nominations de référents nouveaux dans des situations elles-mêmes inédites.

Dans ces conditions une question vient alors à l'esprit: d'où provient la plasticité sémantique de ces items linguistiques qui leur permet d'acquérir des sens aussi divers dans un cadre relativement rigide, au sens où n'importe quel préfixe ou particule ne peut se combiner avec n'importe quel verbe?

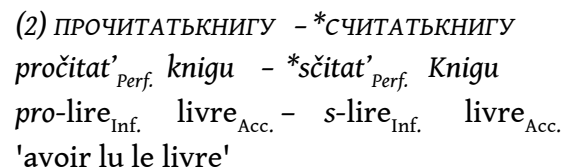

10 Affecté à un verbe,un préfixe ou une particule peut avoir des sens si différents que même les locuteurs natifs ont parfois le sentiment que certains d'entre eux ne sont pas reliés. A titre d'exemple, citons la combinatoire break in. En effet, tandis qu'en (3) elle signifie "mettre ses chaussures jusqu'à ce qu'elles deviennent confortables", en (4) cette même combinatoire désigne "roder une voiture" :

(3) I didn't have time to breakin my new boots, and now I've got blisters all over my feet.

'je n'ai pas eu le temps d'assouplir mes chaussures et maintenant j'ai plein

d'ampoules sur tous mes pieds'

(4) Don't drive the new car too fast, I'm still \{breaking/running\} it in

'ne conduits-pas si vite la nouvelle voiture, je continue à la roder'

11 Il se trouve qu'avec l'hétérogénéité de sens de ces items, il n'est pas aisé non plus de poser une seule unité polysémique et de dégager un invariant sémantique sans aboutir, dans certains cas, à des contradictions non négligeables. En effet, la description du sens en ces termes se heurte au paradoxe d'invariant sémantique: l'invariant se trouve systématiquement soit trop étroit pour englober la totalité des valeurs et des emplois de ces polysèmes, soit trop large pour sélectionner efficacement les valeurs et les emplois dont on cherche à rendre compte.

12 Au problème de l'identité sémantique s'ajoute celui de la polyvalence catégorielle. Alors qu'en russe il s'agit de l'ambivalence préfixe-préposition, certains opérateurs anglais, appelés également marqueurs, peuvent remplir à la fois des fonctions de particule, de 
préposition, de préfixe et d'adverbe. Les énoncés ci-dessous illustrent ces capacités avec OVER :

(5) It takes an experienced politician to get such an unpopular message over

'il faut être un politicien expérimenté pour faire passer un message aussi

impopulaire' (particule)

(6) a. The cover of the book was overlaid with gold and silver (préfixe)

'la couverture du livre est rehaussée d'or et d'argent'

b. ?to lay gold over the cover of the book

'couvrir d'or la couverture du livre'

c. ?The cover of the book was laid with gold and silver /*The cover of the book was laid over

(7) a. Look at the car over there (adverbe) ${ }^{2}$

'regarde la voiture là bas'

b. *Look at the car there over 
crucial dans l'émergence du sens des verbes préfixés ou à particule, d'autant que dans certains cas ni le préfixe ou la particule, ni le verbe avec lequel ils se combinent, n'ont de rapports directs avec la valeur émergée du verbe préfixé ou à particule. Quel est le rapport, par exemple, en russe, entre le verbe non préfixé вить - bit' ("frapper, donner des coups") dans БИТЬПОКАРМАНУ - bit'po karmanu signifiant"coûter cher" et son préfixé РАЗБИТЬ - razbit' ("casser") dans РАЗБИТЬСЛОВАНАСЛОГИ - razbit' slova na slogi signifiant ("découper les mots en syllabes") ?

Faut-il en conclure que lors de la construction d'un sens plus complexe les composants de la combinatoire perdent les éléments de leurs sens 'de base' respectifs au profit d'une nouvelle valeur émergée?

Ce qui nous oblige à nouveau à soulever la question de l'identité sémantique de ces polysèmes.

\section{La réconciliation : une ébauche de solution}

Si l'on veut apporter un début de solution aux problèmes exposés, il semble évident que tant que l'on privilégiera la diversité des effets de sens et d'emplois au détriment de l'unité sémantique et vice versa, au lieu de les concilier, le sentiment que non seulement les différents emplois et valeurs sont liés mais également qu'ils se réfèrent à un système sous-jacent, restera au niveau des intuitions et des appréciations personnelles des linguistes et des locuteurs natifs.

Cette évidence d'une nécessaire réconciliation entre la diversité et l'unité a été, d'ailleurs, à plusieurs reprises soulignée dans la littérature linguistique en question :

[...] the semantic information in a lexical entry for a polysemous item must consist of more than a list of meaning or uses; the specific nature of relatedness between senses must be encoded as information about that item, or about a class of items, (Brugman (1988: ii). Comment concilier [...] la polysémie et la nécessaire stabilité d'un système robuste, qui doit avoir les propriétés de tout système à la fois fermé (d'où du prévisible), et ouvert (d'où des étagements et des déformations qui comportent une part d'imprévisible?, (Culioli (1999: 21).

Ceci entraîne le besoin de poser l'analyse des ces items au niveau d'une plus grande abstraction que celle que fournirait une approche purement sémantique, inapte à établir des liens suffisamment fiables entre les différentes valeurs comme à les déduire à partir d'un invariant.

Si l'approche sémantique montre assez vite ses limites, c'est parce que, dans le cadre de ce modèle, le sens d'un élément linguistique est décrit en termes d'ensemble de conditions nécessaires et suffisantes, partagées par tous les membres d'une catégorie donnée aux frontières strictement délimitées. Cela rend difficile la catégorisation des référents qui ne correspondent pas exactement aux propriétés communes définies pour la catégorie. En fait, selon Niklas-Salminen (1997: 144), la catégorisation d'une forme linguistique étant un des problèmes majeurs des théories sémantiques, a pour origine deux postulats sur lesquels est basée la conception sémantique classique. Selon le premier, les membres d'une catégorie ont un statut égal. Selon le second, les frontières d'une catégorie sont clairement délimitées.

C'est pourquoi, dans la section suivante de cet article, nous proposons de discuter de la description de la sémantique des préfixes et des particules en la situant dans le cadre de 
deux modèles théoriques qui tentent de résoudre les difficultés auxquelles sont confrontées les théories sémantiques classiques en développant d'autres conceptions plus souples du sens des unités linguistiques, à savoir la sémantique du prototype et la sémantique de la Forme Schématique (FS).

\section{Le modèle cognitif et la TOE : deux approches du problème}

31 Appartenant à des courants linguistiques apparemment différents, les deux approches, dont il sera question ici, convergent cependant dans leur ambition de formaliser et de généraliser la thèse selon laquelle les morphèmes polysémiques possèdent une unité sémantique, tout en prenant en compte la diversité de leurs emplois et de leurs valeurs.

Ces deux approches se rejoignent également dans l'idée que cette généralisation ne sera atteinte que par la réconciliation entre la diversité et l'unité et elles proposent des théories qui cherchent à décrire l'organisation interne et externe des items polysémiques en relation avec leur fonctionnalité. L'une opère dans le cadre du modèle cognitif, l'autre se situe dans le cadre de la Théorie des Opérations Enonciatives d'Antoine Culioli (TOE).Cette dernière approche considère qu'il est possible de définir l'identité sémantique d'un polysème. Présente dans tous ses emplois, cette identité sémantique, définie par une Forme Schématique (FS), ne correspond cependant à aucune valeur concrète du polysème dans un contexte donné. On y observe aujourd'hui deux axes de recherche : l'un explore le domaine notionnel dans le champ des particules prépositionnelles et adverbiales de l'anglais (Dufaye 2006, 2009), l'autre, développé dans les travaux de Dobrušina, Mellina, Paillard (2001), Paillard (2004, 2007), Camus (1998), privilégie la description du sens au sein de la Théorie des Opérations Prédicatives et Enonciatives (TOPE). Cette dernière approche sera utilisée dans le cadre de cet article pour exposer les principes théoriques énonciatifs dans le domaine de la préfixation en russe.

Quant au modèle cognitif de Lakoff et Langacker, il est appliqué au domaine de la préfixation du russe par Janda (1986, 1988), Flier $(1975,1985)$, Plungjan (2001) et aux particules prépositionnelles et adverbiales de l'anglais par Brugman (1988), Rudzka-Ostyn (2003), Cappelle \& Declerck (2005), pour ne citer qu'eux. Selon ce modèle théorique, la description de la sémantique d'un item polysémique doit être présentée sous forme d'un réseau structuré des valeurs dont une est centrale ou prototypique.

Outre leurs conceptions distinctes de l'activité langagière, ces points de vue opposés ont pour origine un niveau d'analyse différent auquel les deux modèles se réfèrent respectivement pour rendre compte de la stabilité de l'unité sous-jacente et de la déformabilité des valeurs des items polysémiques. Pour la clarté de notre présentation, nous les avons récapitulés sous forme de Figure 1 :

Fig.1. Modèle Cognitif et la TOE : niveau d'analyse comme une divergence fondamentale

\begin{tabular}{|c|c|}
\hline Modèle Cognitif & Théorie des Opérations Enonciatives \\
\hline $\begin{array}{c}\text { maintien du niveau d'analyse } \\
\text { sémantique }\end{array}$ & $\begin{array}{c}\text { passage au niveau d'analyse } \\
\text { topologique }\end{array}$ \\
\hline
\end{tabular}




\begin{tabular}{|l||ll|}
\hline $\begin{array}{c}\text { renoncement à l'invariant } \\
\text { sémantique }\end{array}$ & $\begin{array}{c}\text { introduction de l'invariant } \\
\text { fonctionnel }\end{array}$ \\
\hline
\end{tabular}

Le modèle cognitif conserve le niveau d'analyse sémantique au détriment de l'invariant. Il constitue son système de représentation du sens d'un polysème à partir des relations hiérarchisées de différentes valeurs par rapport à celle du prototype spatial, cette hiérarchisation étant basée sur la distance sémantique qui sépare ces valeurs du prototype.

Quant au 'prototype spatial', il fait par définition appel à des références concrètes (directions, dimensions, délimitations, etc.), d'où l'importance cruciale de la détermination des critères permettant d'établir sa valeur à partir de laquelle, par extension, sont ensuite obtenues toutes les autres valeurs au moyen des principes cognitifs généraux (métaphore, métonymie, généralisation, spécialisation, transformation d'images schématisées).Selon le cadre théorique cognitif, cette manière de représentation du sens permet à la fois de préserver la diversité de sens et l'unité sémantique sous-jacente des items polysémiques.

A titre d'exemple, la Figure 2 récapitule la configuration prototypique du préfixe verbal russe PERE- et l'opérateur anglais OVER.

Figure 2. Configuration prototypique de PERE-,selon Janda (1988), et OVER, selon Brugman (1988)

\begin{tabular}{|c|c|}
\hline PERE- ("transfer" + “duration") & OVER ("above" + “across") \\
\hline 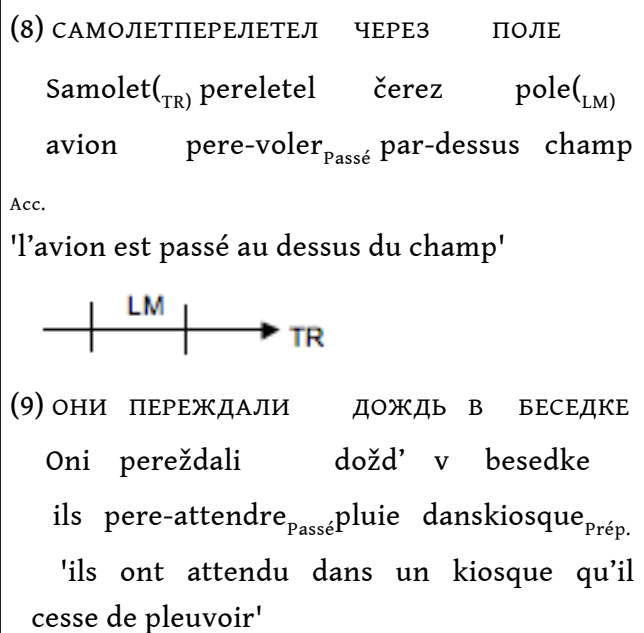 & 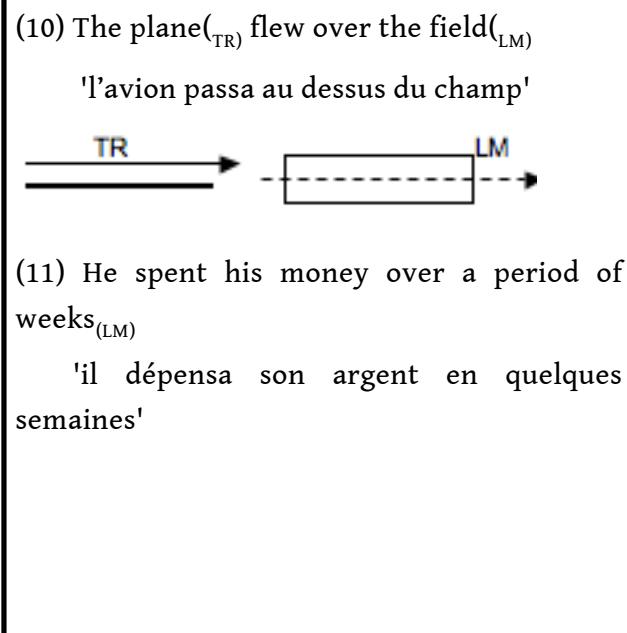 \\
\hline
\end{tabular}

Discutant le positionnement des valeurs du préfixe verbal russe PERE- en termes de centre et de périphérie, Janda (1988 : 339), par exemple, explique la position centrale de la valeur prototypique par deux critères :

- la désinence par le prototype des sens centraux pour un préfixe donné,

- la fréquence de l'utilisation de ces sens par rapport à d'autres considérés comme périphériques du fait de leur moindre fréquence d'utilisation.

Ce type de raisonnement n'a rien de surprenant si l'on prend en considération le fait que le courant cognitif s'intéresse davantage à une description psychologique du référent des 
mots qu'à leur définition. Comme le souligne Niklas-Salminen (1997 : 146), l'une des idées centrales de la sémantique du prototype est que, parmi les stimuli que les gens reçoivent, certains types idéaux ou "prototype" sont des points d'ancrage pour la perception. Ce qui signifie que, d'abord, nous avons affaire à un phénomène plutôt biologique, et, qu'ensuite, la notion de prototype est fondamentalement liée aux individus, qui, en tant que sujets parlants, reconnaissent le meilleur représentant de telle ou telle catégorie.

Cependant, il semble difficile d'estimer fiables les critères faisant appel à des points de vue subjectifs, car dans ce cas cela signifierait que la configuration prototypique varierait d'un individu à l'autre.

41 En effet, sur quels critères objectifs peut-on se fonder pour juger que la fréquence d'utilisation des sens peut mettre un certain nombre d'entre eux à la périphérie sémantique par rapport aux autres significations? Etant russophone d'origine, il nous est difficile d'imaginer, par exemple, que le sens "transfert" représente mieux le sens de PERE- que celui de "division".

En fait, cette importance accordée à la valeur prototypique spatiale a pour origine une autre hypothèse cognitiviste selon laquelle notre perception de l'espace détermine notre capacité d'abstraction.

Etant donné que, selon cette hypothèse, les images schématisées structurent notre expérience physique, au sens le plus large, mais également toute autre forme d'expérience au moyen de métaphores, ces images schématisées peuvent être utilisées pour décrire la conceptualisation d'expériences, autrement dit la transformation symbolique en concepts des éléments de la réalité ou de toute forme d'expérience.

Cette hypothèse constitue une autre opposition fondamentale entre les deux modèles en question.

En effet, selon la TOE, c'est notre mode d'abstraction qui détermine l'organisation de nos perceptions, spatiales en l'occurrence (Dufaye 2006 : 171). Et même si les représentations spatiales sont primaires dans notre activité consciente, elles ne révèlent, ainsi que toute autre représentation, qu'une activité cognitive liée aux opérations mentales auxquelles les linguistes n'ont d'accès qu'à travers l'empirique. Il s'en suit que, si les autres valeurs d'une unité polysémique peuvent être attachées à la valeur prototypique spatiale, c'est plutôt par analogie que par extension.

Une autre conséquence de cette hypothèse énonciativiste est que l'on ne peut pas traiter le système des préfixes et des particules prépositionnelles et adverbiales au moyen d'outils géométriques, les images schématisées en l'occurrence.

D'ailleurs les cognitivistes eux-mêmes reconnaissent cette fragilité de leur représentation. Ainsi, pour Brugman (1988:3), auteur du protopype de OVER:

[...] imaginal representations of the spatial relationship are not identical with the mental representations, the nature of which I can make no claims about. However, since the depictions exploit familiar spatial configurations existing in our experience of the world [...] I do believe that the depictions bear some resemblance to the corresponding mental representations, [...].

La manière dont le cerveau rend possible l'expérience humaine consciente et sa représentation reste encore mystérieuse.

Le passage au niveau d'analyse topologique, au contraire, permet à la TOE de recourir à des abstractions topologiques généralisables à tout un ensemble de valeurs et, par 
conséquence, de proposer l'invariant fonctionnel sous forme schématique abstraite couvrant les emplois non hiérarchisés d'un item polysémique.

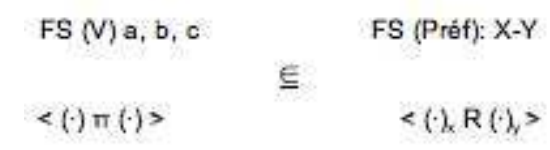
fonctionnel couvrant les emplois non hiérarchisés de l'élément décrit. Ce que Camus (1998 : 103) résume parfaitement ainsi : "Dans ce cadre, l'invariant constitue un potentiel de variation". Ceci revient à dire que la FS est apte à rendre compte de tous les sens possibles sans être un des sens concrets de l'élément décrit. ${ }^{3}$

Le second niveau représente l'ensemble des variations régulières régissant la combinatoire de l'élément décrit avec le cotexte dans lequel il apparait et fait reposer la construction du sens de la combinatoire Préf-V sur une relation de repérage entre le V, élément repéré4 , et le Préf, élément repère.

Ainsi, pour ce qui concerne les verbes préfixés, par relation de repérage (marquée sous forme d'un epsilon miroir dans la figure ci-dessous) il faut entendre la reconstruction d'un des composants sémantiques de la FS (V) dans l'espace sémantique du Préf.

Figure 3. Combinatoire Préf-V et la relation de repérage

En fait, selon la TOPE, les relations de repérage s'affranchissent des frontières des lexèmes. Constituée par l'ensemble des repérages mis en jeu, une FS est ensuite associée à un lexème donné. Comme le souligne Paillard (1992: 81), cette FS fera l'objet de déformations en fonction de déterminations spécifiques, donnant lieu aux 'valeurs locales' de l'item en question.

Constatons ici que la sémantique du prototype présente une certaine similitude avec une FS, dans la mesure où elle cherche également à attribuer au sens d'un polysème une plasticité aux frontières relativement floues.

Cependant, tandis qu'établie au niveau topologique, une FS, quelle que soit la déformation à laquelle elle est soumise, demeure toujours intacte, le prototype, par contre, basé sur un sens concret est par conséquent modifiable par des déformations métaphoriques du sens.

Selon Paillard (2007: 140), dans la Figure 3, a, b, c, composants sémantiques de la FS (V), occupent une place d'argument variable dans le schéma prédicatif. Ils entrent dans des relations, définies par la FS, les uns avec les autres. Toutefois a n'est jamais la base verbale $\mathrm{V}$. Associés de façon stable à une place d'argument de la relation prédicative, $\mathrm{X}$ et $\mathrm{Y}$ sont les deux entités mises en relation par le Préf. Autrement dit, un élément $a$ de la FS (V), qui n'est jamais la base verbale en tant que telle, est pris comme $\mathrm{X}$ dans l'espace sémantique $\mathrm{du}$ Préf où il reçoit les déterminations dont $\mathrm{Y}$ est la source. Ceci signifie que la reconstruction d'un élément $a$ de la FS (V) présente une double opération :

1) l'élément $a$ de la FS (V) est pris comme $X$ dans l'espace sémantique du Préf

2) $a$ en tant que $X$ reçoit les déterminations dont $Y$ est la source (à la différence de $X, Y$ n'a pas a priori de rapport direct avec un élément de la FS (V). 
60 Ainsi, l'identité sémantique de PERE- peut être décrite de la façon suivante : l'événement pris en charge par PERE- est lié à la notion de 'passage' du terme $\mathrm{X}$ d'un état $\mathrm{e}_{\mathrm{i}}$ (Sit1) à un état $e_{j}$ (Sit2) où $e_{i}$ et $e_{j}$ sont deux états quantitativement et/ou qualitativement distincts (il y a discontinuité entre les deux états) ${ }^{5}$. Lié à la notion de 'passage', Y est un domaine topologique de PERE- où sont définis $\mathrm{e}_{\mathrm{i}}(\mathrm{Sit} 1)$ et $\mathrm{e}_{\mathrm{j}}(\mathrm{Sit} 2)$ du terme $\mathrm{X}$. Il est important de signaler que la définition par Paillard (2002: 170) de Y comme domaine topologique permet d'y distinguer différentes zones d'altération (intérieur, extérieur, frontière, etc.). Il s'en suit que l'altérité Sit1/Sit2 sur le domaine Y relève de l'altérité des zones.

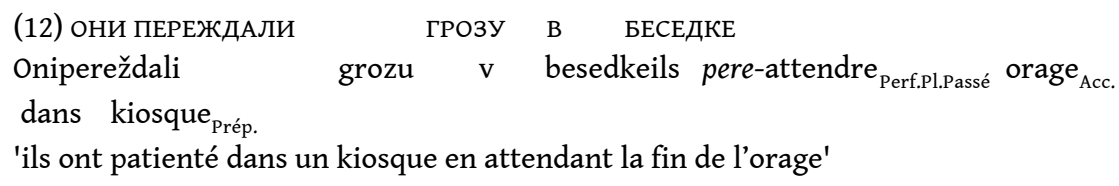

61 En (12), X, qui correspond à ГРОзу - grozu ("orage"), est pris en charge par PERE- en tant qu'événement qui passe de son état initial $\mathrm{e}_{\mathrm{i}}(\mathrm{Sit} 1)$ - "début de l'orage" à son état final $\mathrm{e}_{\mathrm{j}}$ (Sit2) - "fin de l'orage". Le procès désigné par la base verbales жДАТь - ždat' ("attendre") n'est que le moyen pour passer de l'état $e_{i}$ (Sit1) à l'état $e_{j}$ (Sit2).

62 Egalement conditionnée par leurs hypothèses fondamentales respectives, l'activité langagière, considérée du point de vue cognitif comme une activité de perception et de production des séquences temporelles des unités symboliques, discrètes et structurées, implique, selon la TOE, la reconnaissance et la production des formes empiriques, ces formes étant à la fois la trace des représentations mentales et la base de la construction des représentations métalinguistiques.

63 L'approche méthodologique qu'implique cette dernière manière de concevoir le langage nécessite un va-et-vient permanent entre l'observation et la théorisation des phénomènes linguistiques étudiés. L'objectif essentiel d'une telle démarche est, à partir de l'empirique et au moyen de procédures canoniques d'abstraction et de formalisation, la construction d'une métalangue de représentation stable et cohérente, libre de toute influence subjective.

\section{En guise de conclusion}

64 Ainsi, nous dirons que malgré les divergences que nous avons cherché à mettre en évidence tout au long de cette étude, ces deux approches semblent apporter des éléments nouveaux qui rendent de grands services à la sémantique lexicale, au moins à deux égards.

Pour ce qui nous intéresse, elles prouvent, d'abord, l'existence d'une organisation interne à l'intérieur d'une unité linguistique, y compris polysémique. La fixité centrale d'une configuration prototypique au sein du modèle cognitif et l'organisateur à l'intérieur du domaine notionnel dans le cadre de la TOE apportent par leur permanence relative la stabilité structurale nécessaire à l'unité d'un item.

Elles permettent, ensuite, chacune à sa manière, de réintégrer dans le sens d'un polysème, des significations hautement hétérogènes sans perturber l'unité sous-jacente de celui-ci.

Cependant, malgré ces avantages qui font progresser l'analyse sémantique des unités polysémiques, les deux modèles en question ne constituent pas une panacée. Comme tout autre modèle théorique, ils se heurtent à des obstacles qu'ils ont du mal à surmonter. 
Le modèle cognitif garde le niveau d'analyse sémantique au détriment de l'invariant et constitue son système de représentation du sens sur les relations hiérarchisées entre différentes valeurs d'un polysème, ce qui favorise en fin de compte sa division et ramène à une description autonome de chacune de leur signification.

assage au niveau d'analyse topologique permet à la TOE de garder l'invariant sous forme schématique abstraite couvrant les emplois non hiérarchisés d'un item et donc de préserver son unité. En même temps, le haut niveau d'abstraction qui caractérise toute forme schématique présente un obstacle quant à son application.

Ainsi, deux types de représentation du sens s'offrent au linguiste en fonction du point de départ choisi. Ceci oblige chacun à faire son propre choix entre ces deux niveaux d'analyse et par conséquent entre ces deux modèles pour traiter les unités polysémiques, ce qui n'exclue en aucun cas l'existence éventuelle d'autres hypothèses de départ permettant l'élaboration de nouveaux modèles de la représentation du sens et la possible émergence d'approches alternatives de la polysémie.

\section{BIBLIOGRAPHIE}

Brugman, C. M. (1988) : The story of over : polysemy, semantics and the structure of the lexicon. New York, London (Garland).

Camus, R. (1998) : Quelques considérations sur le préverbe po- en russe contemporain. In : Revue desétudes slaves LXX/1. Paris (Institut d'Études Slaves), 101-112.

Cappelle, B., Declerck, R. (2005) : Spatial and temporal boundedness in English motion events. In : Journal of Pragmatics 37, 889-917.

Culioli, A. (1999) : Pour une linguistique de l'énonciation. Tome 3. Paris (Ophrys).

Culioli, A. (2000) : Pour une linguistique de l'énonciation. Tome 1. Paris (Ophrys).

Dobrušina, E., Mellina, E., Paillard, D. (2001) : Russkie pristavki : mnogoznačnost' i semantičeskoe edinstvo. (Les préfixes russes : polysémie et identité sémantique). Moskva (Russkie slovari).

Dufaye, L. (2006) : OFF and ON : Projet de représentation formelle. In : Souesme, J.-C.(ed.) :

CYCNOS, Le Qualitatif. Nice (CRELA), 171-187.

Dufaye, L. (2009) : Théorie des opérations énonciatives et modélisation. Paris (Ophrys).

Flier, M. S. (1975) : Remarks on Russian Verbal Prefixation. In : Slavic and East European Journal $19,2,218-229$.

Flier, M. S. ([1985]1997) : The scope of préfixal delimitation in Russian. In : Krongaouz, M. (ed.) : Glagol'naja prefiksacija v russkom jazyke (La préfixation verbale en russe). Moskva (RSl), 29-48.

Franckel, J.-J. (2006) : Situation, contexte et valeur référentielle. In : Textes, contextes, Pratiques, CRESEF 129-130. Université de Metz, 51-70. Version électronique, 1-19.

Gettliffe, P. (1999) : Verbes prépositionnelles et verbes à particules. Paris (Ellipses). 
Janda, L. A. (1986) : A Semantic Analysis of the Russian Verbal Prefixes za-, pere-, do-, and ot-. München (Verlag Otto Sagner).

Janda, L. A. (1988) : The Mapping of Elements of Cognitive Space onto Grammatical Relations : An Example from Russian Verbal Prefixation. In : Rudzka-Ostyn, B. (ed.) : Topics in Cognitive Linguistics. Amsterdam (John Benjamins), 327-343.

Johnson, M. (1987) : The Body in the Mind : The Bodily Basis of Meaning, Imagination, and Reason. Chicago, London (University of Chicago Press).

Lakoff, G., Johnson, M. (1980) : Metaphors We Live By. Chicago, London (University of Chicago Press).

Lakoff, G. (1986) : Cognitive Semantics. Berkeley (University of California).

Langacker, R. (1987) : Foundations of Cognitive GrammarI. Stanford : (University Press).

Langacker, R. (2002) : Concept, image and symbol. New York (Mouton de Gruyter).

Niklas-Salminen, A. (1997) : La lexicologie. Paris (Armand Colin).

Rudzka-Ostyn, B. (2003) : Word Power : Phrasal Verbs and Compounds. A Cognitive Approach.New York (Mouton de Gruyter).

Paillard, D. (2004) : A propos des verbes préfixés. Slovo 30-31, 13-44. Version électronique, 1-23.

Paillard, D. (2007) : Verbes préfixés et "intensité" en français et en russe. In : Travaux de Linguistique 55, 133-149.

Plungjan, V. (2001) : Pristavka pod- v russkom jazyke : k opisaniju semantičeskoi seti (Le préfixe pod- en russe : à propos du réseau sémantique de valeurs). In : Krongauz M. (ed.) : Glagol'nye prefiksy et prefiksal'nye glagoly 1 (Les préfixes verbaux et les verbes préfixés). Moskva (Moscow Journal of Linguistics), 95-124.

Slovar' sovremennogo russkogo literaturnogo jazyka (Dictionnaire du russe moderne), (1961). T. 1-17. Moskva, Leningrad (Izd-vo AN SSSR).

The Oxford English Dictionary, ([1933]1961). Oxford (At the Clarendon Press).

\section{NOTES}

1. Cf., l'article «Rostovski opyt kreditovanija malogo biznesa» (L'expérience de Rostov en matière d'octroi de crédit aux petites entreprises), dans l'hebdomadaire Novosti malogo biznesa (Les actualités des petites entreprises), version électronique 15(157) du 19.04.2002.

2. L'exemple (7) est emprunté à Gettliffe (1999: 8).

3. Cf., par ex., Franckel (2006: 15): "Une FS définit l'identité d'un mot comme la façon particulière qu'elle a d'incorporer et de construire les environnements qu'elle rend possible".

4. Cf. Culioli (2000 : 116) : "Tout objet [...] est toujours pris dans une relation : il n'y a pas d'objet isolé. C'est précisément ce que veut dire repéré".

5. Cf. Paillard (2004:21, $2007: 144)$. 


\section{RÉSUMÉS}

Le présent article se propose, à partir des préfixes verbaux russes et des particules verbales anglaises, de discuter de la description de la sémantique des morphèmes polysémiques en les situant dans le cadre des deux approches appartenant à des courants linguistiques apparemment différents : l'une opère dans le cadre du modèle cognitif, l'autre se situe dans le cadre théorique des Opérations Enonciatives d'Antoine Culioli. La comparaison des deux cadres théorique sera effectuée afin de démontrer qu'ils convergent dans leur ambition de formaliser et de généraliser la thèse selon laquelle les morphèmes polysémiques possèdent une unité sémantique, tout en prenant en compte la diversité de leurs emplois et de leurs valeurs.

The present paper is concerned with the phenomenon of polysemy. Specifically, the article deals with such polysemes as verbal prefixes in Russian and verbal particles in English and discusses their semantic identity from two different theoretical perspectives: Cognitive Linguistics and Culioli's Theory of Enunciative Operations (T.E.O). We will compare the framework of cognitive linguistics with the framework of T.E.O in order to show to which extent these two seemingly opposed theoretical approaches coincide in their efforts to come to grips with polysemy.

INDEX

Keywords : polysemy, semantic identity, verbal particles, verbal prefixes

Mots-clés : identité sémantique, particules verbales, polysémie, préfixes verbaux

\section{AUTEUR}

OLGA KRAVCHENKO-BIBERSON

INALCO 